ty of beryllium gives us hope that toxicity will not forever be a barrier to the wider use of this metal and alloys containing large amounts of it. The reason why only $2 \%$ of human beings are sensitive to beryllium may be hereditary. Beryllium dust in the lungs of sensitive people causes their immune systems to try to isolate it from the rest of the body. Over a period of many years, this causes serious damage to the lungs. Various medical tests can now identify people who are sensitive to beryllium. However, for some of these tests to work, it is necessary that the person already have sustained beryllium exposure. If research could identify the hereditary factor, the sensitivity tests could be performed on individuals before they are exposed to beryllium dust. It is a sad fact that today not enough money is being invested in the medical research that would lead to the answers that are needed.

Finally, this third problem with beryllium application is made more complicated because we live in a period of time that demonstrates a great aversion to taking any risk, and with widespread litigation to pin down responsibility on someone other than the injured party. Risks about which little is known are greatly feared even though the actual risk is far less than such a well-known and widely accepted risk as driving to work. Beryllium dust is very hard to contain and hard to find; this along with the misconception about beryllium toxicity makes it seem very risky, indeed, to use. Our laboratory and workplace procedures are now becoming very strict regarding beryllium operations, and so our workers are much less at risk for inhaling beryllium dust and possibly con- tracting chronic beryllium disease.

So, let's go back to the positive aspect of beryllium: its promise as a "stairway," the structural metal that we need in order to explore new frontiers. When your mother was your age, the U.S. Air Force embarked upon a major effort to develop a new class of structural materials based on high stiffness fibers embedded in a plastic matrix. Back then, these materials were believed to be the answer to just about every aerospace structural materials problem. These new "advanced composite" materials received a very large amount of research and development investment, and today they make important contributions to the performance of structures ranging from spacecraft to sporting goods.

I have no doubt that beryllium could make at least equal, and probably greater, improvements in structural performance, particularly when incorporated into other metals such as aluminum with which it forms a very interesting and useful composite structure. The plastics from which advanced composites are made are themselves highly toxic substances. Advanced composite materials are not nearly so resistant to high temperatures, radiation, and the rigors of high vacuum environments in space. So beryllium, and its composite with aluminum, are excellent choices for spacecraft structure. Beryllium-aluminum material is also being tested today for pos-

Material Matters is a forum for expressing personal points of view on issues of interest to the materials community. sible use as an aircraft control surface, and if successful, this material could lead to significant savings in the weight of future aircraft structures.

I've had the very fortunate experience of meeting metallurgists who specialize in beryllium from all countries of the world where this metal is produced. We all share a common hope that beryllium, a metal which was developed in the past as a material of war, will in the future make important contributions to peaceful applications. All people should be able to realize a significant return on the past investments that were made to develop beryllium metal for its military uses. More efficient structures will result in huge energy savings for transportation systems, particularly those that your generation will be using for space travel. Many other materials would be significantly improved with the addition of beryllium. Perhaps the key issue right now is the lack of progress on the health research that could one day eliminate the danger of beryllium toxicity. I hope that by the time you are able to read this letter all by yourself, this issue will be well on the way to being resolved.

Love,

\section{Grandpa Jake}

Loren A. Jacobson is a technical staff member in the Metallurgy Group, Materials Science and Technology Division, Los Alamos National Laboratory, Los Alamos, New Mexico, USA. The opinions presented here are those of the author, and do not represent official positions of Los Alamos National Laboratory, the University of California, or the U.S. Department of Energy.

\section{Assistance of AFM Produces Tiny Transistor}

Making simple and elegant use of a chemical theory of liquids, scientists at Weizmann Institute have developed a way to predict the minimal possible size of bipolar transistors, one of the major types of transistors commonly used in microelectronics. Using the experimental semiconductor copper indium diselenide, they have manufactured such a structure. With an inner core of $20 \mathrm{~nm}$ and total width of $50 \mathrm{~nm}$, the device is five times smaller than the otherwise smallest standard transistors of this type.

For this research, reported in Applied
Physics Letters, doctoral student Shachar Richter used atomic force microscopy to manipulate atoms in a semiconductor. Normally, such microscopes can only shift atoms on the surface of a material, but Richter, building on earlier research by David Cahen of the Materials and Interfaces Department, moved these atoms around inside the semiconductor.

Richter achieved his results by applying a voltage to the semiconductor and passing a current through the material. Aided by the slight heating produced by the current, the voltage caused dopants to be propelled in a particular direction. Even though only 100-200 dopants were moved in this manner, this sufficed to produce a tiny transistor. It consisted of a hemispherical layer of relatively high conductivity containing the redistributed dopants, flanked on both sides by material with different conductivity.

Richter next used the same microscope stylus-at low voltage-to map the conductivity of this miniature structure. Richter's new mapping method, called scanning spreading resistance, reveals the precise path that would be taken by an electric current flowing through a transistor of this type. Richter said that this device may serve as a valuable research tool for studying the limits of miniaturization. 


\section{Discovery of Nanospheres 18-nm in Diameter Brings Researchers Closer to Synthesizing Tooth Enamel}

Researchers at the University of Southern California's School of Dentistry are closing in on making tooth enamel, the hardest substance found in vertebrates. They have identified tiny spheres that regulate the formation and organization of tooth enamel by controlling the substance's crystalline growth. While the researchers first identified the crystalline spheres in 1994 as magnified in an electron microscope, an atomic force microscope recently revealed that the spheres are uniformly $18-20 \mathrm{~nm}$ in diameter. Called nanospheres, these structures are formed by a naturally occurring family of toothspecific proteins known as amelogenins.

Chemically, the mineral crystals in tooth enamel are a calcium hydroxyapatite formed from calcium and phosphate ions, which are transported into the nanosphere matrix by ameloblast cells.

Research professor A.G. Fincham said, "At first, the elongated apatite crystals will grow solely on their end faces, becoming ever longer. With the nanospheres acting as spacers, these early crystals build a scaffold on which mature enamel can eventually form. After enzymes have broken down the amelogenin proteins, the crystals start to grow on all of their faces. They thicken, clump together, and create mature enamel."

Apatite crystals grown in the laboratory by traditional methods that rely on precipitation of the mineral from solutions of calcium and phosphate ions are about 100 times smaller than the crystals nature makes. They grow haphazardly, and the resulting material is considerably weaker than natural enamel.

Four years ago, the researchers took the gene for an amelogenin protein from a mouse, placed it in a bacterial cell, and then used the bacterial reproductive process to produce an identical recombinant amelogenin protein. This recombinant amelogenin protein, which the researchers can now produce in quantity, has since been shown to self-assemble to make nanosphere structures identical to those seen in the mouse and other animals, including humans.

Fincham said that the function of the amelogenin enamel protein "is to spontaneously self-assemble into a matrix with nanospheres-a matrix that controls the microarchitecture of the developing enamel, both the three-dimensional spacing between the initial mineral crystals and the later crystal growth."
Currently, the researchers are growing apatite crystals within synthetic matrices made from recombinant amelogenin protein. They form a gel matrix by the sedimentation (centrifugation) of amelogenin protein precipitated from solution into a small glass open-ended tube which is then inserted into a "gel double diffusion" apparatus in which calcium and phosphate ions diffuse into the gel from opposite sides. The mineral is formed within the gel matrix where the ions and the effects of the protein matrix on the size, orientation, and form of the crystals can be studied. The researchers report their work on the structure of the nanosphere-gel matrices in an upcoming issue of the Journal of Structural Biology.

Fincham said, "We get very long, straight structures. The crystals grow only on their end faces, although the material is unremarkable. We can't make enamel yet, but we can see how nature does it. The nanospheres clearly have a capacity to regulate the way crystals grow."

For two decades, researchers at USC School for Dentistry have been studying tooth enamel with the goal of one day replacing mercury-based gold and silver fillings with restorations of synthetic material identical or similar to natural tooth enamel. Their recent publications include Calcif. Tissue Intl. 38 (1998) p. 237 and J. Struct. Biol. 115 (1995) p. 50.

\section{Robert Baboian Receives National Materials Advancement Award}

Robert Baboian, a consultant in Greenville, Rhode Island, has received the Federation of Materials Societies' 1998 National Materials Advancement Award which recognizes individuals who have demonstrated their outstanding capabilities in advancing the effective and economic use of materials and the multidisciplinary field of materials science and engineering generally, and who contribute to the application of the materials profession to national problems and policy. Baboian has been dedicated to the research and development of corrosion resistant materials and devices for over 35 years, sharing his expertise in a broad range of activities and on a number of government and academic committees. From 1983 to 1986, his services as a corrosion consultant were donated to the National Park Service on the Statue of Liberty restoration program.

After receiving his PhD degree in physical chemistry from Rennssalaer Polytechnical Institute in 1964, Baboian did postdoctoral research at the University of
Toronto and subsequently received a faculty appointment to senior research associate. In 1966 he joined Texas Instruments as a member of the technical staff and established the TI Electrochemical and Corrosion Laboratory in 1968. He was elected a TI Fellow in 1976, a TI Senior Fellow in 1980, and TI Principal Fellow in 1988.

Recognized for his active involvement in the technical community, Baboian has received various honors including the NACE International Speller Award for outstanding contributions in corrosion engineering, the ASTM LaQue Award, the Science and Technology Award of the Suga Weathering Technology Foundation in Japan, the Cavanaugh Award and the Award of Merit from ASTM, and the Vittorio de Nora Award from The Electrochemical Society. He is an ASTM Honorary Member; a Fellow of ASTM, NACE International, SAE; and was a Ford Foundation Postdoctoral Fellow. Baboian has served on the Executive Committee and the Board of Directors of ASTM, FMS, and NACE International, and as chair of the ASTM Board of Directors.

\section{Ames Lab Researchers Receive DOE Award for Work with Quasicrystals}

A team of scientists at Ames Laboratory have received the Department of Energy's 1998 Materials Sciences Award for "Outstanding Scientific Accomplishment in Materials Chemistry" for its work in understanding the surface properties of quasicrystals. The team includes Pat Thiel (director of the Lab's Materials Chemistry Program), James Anderegg, Matt Besser Tamara Bloomer, Alan Goldman, Cynthia Jenks, Matt Kramer, Tom Lograsso, and Dan Sordelet. Also collaborating with the group are Martin Gierer from Ludwig Maximilians University in Munich, Germany, and Michel Van Hove of Lawrence Berkeley National Laboratory.

Quasicrystals were discovered in 1982 and first reported in 1984, changing the long-held belief that solid matter existed in only two states: amorphous or crystalline. Quasicrystal materials fall outside these groups, containing atoms that are arranged in an ordered but not periodic way. In the late $1980 \mathrm{~s}$, scientists discovered that quasicrystals were highly resistant to wear and corrosion, and that they had low coefficients of friction-highly desirable properties for coatings on mechanical and automotive parts. Such coatings would make the parts less vulnerable to wear- and rust-related damage.

What was lacking was an understanding of why quasicrystal materials exhibit- 
ed these surface properties. Thiel said that the Ames group has begun to lay a foundation for understanding this topic.

According to Thiel, approaching quasicrystal research from a variety of disciplines has enabled the group to address not only the fundamental nature of the surfaces of these materials, but also how the materials could be adapted for use in industrial settings.

Materials scientist Dan Sordelet said that the broad-based approach has given the group a more complete understanding of how quasicrystal materials can be used in both consumer and industrial products. "The first application of quasicrystals is as a surface coating for certain frying pans, which are now commercially available," Sordelet said. "We expect our integrated work to lead to other commercial applications ranging from thermal protection of aircraft engines to wearresistant, reduced-adhesion surfaces for injection-molding dies."

The Materials Sciences Awards are given annually to recognize scientists at Department of Energy laboratories for outstanding research.

\section{Pinholes Reduce Density of Threading Dislocations in Strain Relaxed Film}

Based on their research reported in the January 18 issue of Journal of Applied Physics, researchers at Max-Planck-Institut für Mikrostrukturphysik suggest that lateral limitation of the epitaxial grain growth on a thin twisted GaAs layer caused by the presence of pinholes reduces the density of threading dislocations in the strain relaxed film and may be a mechanism for low density of threading dislocations in lattice mismatched epitaxial films grown on twist bonded "compliant universal substrates."

Theoretically, a free-standing epitaxial substrate equal or thinner than the critical thickness with regard to the deposited film should be able to absorb the overall strain energy, regardless of the thickness of the deposited film, without generating any misfit dislocations (i.e., compliant universal substrate). S. Senz and co-workers at MPI, in studying the underlying mechanisms of dislocation reduction, used metal organic vapor phase epitaxy (MOVPE) to deposit a $100 \mathrm{~nm}$ AlAs release layer and $10 \mathrm{~nm}$ of
GaAs on $75-\mathrm{mm}$ diameter (100) GaAs wafers with a twist angle of $14^{\circ}$. After lifting the intermediate AlAs release layer, the researchers used $x$-ray diffraction to determine the coverage of the handling wafer by the bonded thin layer. The investigators report that tunneling electron microscopy "revealed precipitates or voids at the bonding interface, which in most cases do not thread through the GaAs compliant layer. Plan view TEM shows the screw dislocation network and Moiré contrast associated with the bonding interface as well as precipitates/voids." They reported that occasionally the height of the precipitates surpasses the thickness of the GaAs compliant layer and penetrates through the surface. When the researchers deposited $300 \mathrm{~nm}$ of $\operatorname{InP}(3.5 \% \mathrm{misfit})$ or $\operatorname{In}_{x} \mathrm{Ga}_{1-x} \mathrm{As}(x=0.28,2 \%$ misfit) on the GaAs layer at the same twist angle, they identified two different crystallographic orientations of InGaAs grains, twisted and untwisted with respect to the GaAs handling wafer. They observed that the twisted and untwisted grains are separated by tilt grain boundaries and determined that the pinholes in the twist bonded layers are responsible for the occurrence

\section{Excellence....magnified}

Recognized world-wide for maintaining the highest standards of quality and innovation in the industry

TEM SPECIMEN PREPARATION

Ion Mill, Twin-Jet Electropolisher,

Ultrasonic Disk Cutter,

Disk Punch, Dimpling Grinder,

Cross-Section TEM Prep Kit

PLASMA CLEANERS

For the simultaneous removal of organic contamination from TEM and SEM specimens and specimen holders

SPECIMEN HOLDERS

Single tilt rotate, Double tilt analytical, Cryo rotate, Double tilt cryo,

Cryotransfer systems

IMAGING

A creative new force

in the field of imaging
E.A. Fischione Instruments, Inc. 9003 Corporate Circle Export, PA 15632 USA
Tel. 724.325.5444

Fax. 724.325.5443

www.fischione.com

\section{N S T R U M E N T S}

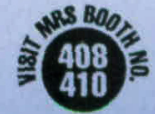

Circle No. 9 on Reader Service Card. 
of tilt grain boundaries.

The researchers report that the "threading dislocation segments, which glide in a direction parallel to the interface, accumulate at the grain boundaries and lower the density of threading dislocation in the epitaxial film."

\section{FMS Honors Robert A. Laudise Posthumously}

In December 1998, the Federation of Materials Societies honored Robert A. Laudise (1930-1998) posthumously with its first Past Presidents' Award for Excellence in Leadership in the Materials Professional Community. Laudise, FMS President in 1995-1996, has been honored "for his long time leadership of strengthening the professional societies in expanding their horizons for greater service to the materials field."

Hailed by the National Academy of Engineering as "a pioneering materials engineer and scientist whose work profoundly influenced the development of lasers, transistors, and other microelectronic devices," Laudise spent his professional life at Bell Labs. At the time of his death he was adjunct chemical director at Bell Labs, adjunct professor of materials science at the Massachusetts Institute of Technology, and adjunct professor of ceramics at Rutgers University.

Laudise was an early and staunch advocate of corporate environmental responsibility, playing a key role in bringing the field of industrial ecology to the attention of the U.S. scientific and engineering communities. His numerous prizes and awards include the American Chemical Society Materials Chemistry Prize, the International Crystal Growth Prize, the Orton Award of the American Ceramic Society, and the Sawyer Prize. The International Organization for Crystal Growth has designated its prize for experimental crystal growth the Laudise Prize.

Laudise served as editor-in-chief of the Materials Research Society's Journal of Materials Research.

\section{Glue Consisting of Silicon- Bearing Polymers and Aluminum-Silicon Alloy Powder Binds Silicon-Carbide Composite Fibers}

Until now manufacturers have been unable to make complex objects-such as fans, heat exchangers, engine parts, and fuel cells-out of composites made of silicon-carbide fibers because reliable joints between parts made of the material could not be formed. Researchers at
Ames Laboratory have developed a glue to solve this problem.

Ames Lab chemist Sina Maghsoodi and three colleagues have developed the pastelike glue made of silicon-bearing polymers and an aluminum-silicon alloy powder. When heated to about $500^{\circ} \mathrm{C}$ the polymers in the glue begin to break down into silicon carbide and excess carbon. As the heat reaches $600^{\circ} \mathrm{C}$, the alloy powder starts to melt. Silicon from the alloy reacts with the excess carbon to form more silicon carbide while the aluminum reacts with available oxygen to form aluminum oxide. These additional ceramic particles, or whiskers, diffuse to strengthen the joint in the same way that the fibers toughen the composite.

Maghsoodi said, "The glue forms an in situ composite that gives strength to the joint area."

The problem of finding a silicon-carbide joining process has been difficult to solve because, although silicon carbide itself can withstand temperatures of up to $2000^{\circ} \mathrm{C}$, the fibers in the composite material begin to degrade at about $1200^{\circ} \mathrm{C}$. Traditional ceramic-joining methods do not work with the composites because they involve curing the joints at temperatures of at least $1600^{\circ} \mathrm{C}$.

The researchers' tests indicate that ceramic joints made with the glue exhibit strength of up to 14,500 pounds per square inch (the equivalent of 100 megapascals) at temperatures of $1200^{\circ} \mathrm{C}$, which is a vast improvement over steel, which has little strength at temperatures above $700^{\circ} \mathrm{C}$.

Maghsoodi said that because the joint can be made by propane-torch heating, manufacturers could use the glue in a variety of field applications rather than having to cure the joints in high-temperature furnaces.

\section{Nonlinear Acoustical Measurements Detect \\ Materials Damage}

Researchers at Los Alamos National Laboratory are measuring nonlinear properties of materials to demonstrate a difference between damaged and undamaged concrete. The research, reported during the fall meeting in December 1998 of the American Geophysical Union in San Francisco, may have broad application for the detection of damage in many materials. Nonlinear materials research includes a technique called nonlinear resonant ultrasound spectroscopy (NRUS), which is being used by graduate student Loren Byers, also of the Geoengineering Group, to measure the difference in the response to an acoustic wave by damaged and undamaged concrete core samples.

In his research using NRUS, Byers imparts energy to a concrete sample through a speaker-like device that sends a sound wave through the sample. The speaker is set up to input tones over a range from below to above the sample's resonant frequency. At each tone in the range, he measures the volume and frequency of the wave at the other end of the sample. This procedure is used at progressively increasing volume levels.

In a sample that is undamaged, the volume output is directly related to the volume input, and therefore the resonant frequency remains the same as the applied volume is increased. In a sample that is damaged, the resonant frequency shifts as the applied volume increases, and the amount of the shift can be measured precisely.

Byers said that the frequency shift is greater in samples that are more damaged, even with microscopic cracks. So, for example, in a piece of plastic that contains no cracks, there will be no change in resonant frequency as the wave volume is increased. In a sample with a small crack, the resonant frequency will shift readily with amplitude.

Concrete is always slightly damaged in the curing process due to pressures from chemical reactions that build up inside and crack the sample. In a fresh concrete core, the resonant frequency shift is measurable, but becomes considerably larger for the damaged sample, with the same volume input. Byers said, "This change makes it very obvious what is damaged and what is fresh concrete. Nonlinearity is extremely sensitive to damage."

Evidence of damage is not only provided by the amount of the frequency shift, but by other manifestations of nonlinearity. For example, Byers also tests samples in a "conditioning and recovery" mode in which a loud-volume tonal sweep is followed by several soft-volume tonal sweeps. In damaged materials it takes some period of time for the sample to return to its original resonant frequency. Furthermore, more damaged samples take longer to return to the original resonant frequency.

Byers said, "Traditionally, nonlinear properties have been ignored in favor of studying the linear properties of the material to detect damage or flaws. Nonlinearity is a new frontier in damage diagnostics."

Paul Johnson of the Geoengineering Group said that the potential uses of nonlinear techniques to reveal defects in materials include inspections of aging aircraft, quality control for assembly line applications, monitoring containment walls of nuclear reactors and applications to the national infrastructure such as examining bridge pillars and other structures. 